Thatiana Lamerka Maciel Amaral ${ }^{1}$

Gina ToRRES Rego MONTERO ${ }^{2}$

\section{Artigo Original}

Palavras-chave

Gravidez

Comportamento sexual Adaptação transcultural

Estudos de validação

Questionários

Keywords

Pregnancy

Sexual behavior

Cross-cultural adaptation

Validation studies

Questionnaires

\title{
Tradução e validação de questionário de função sexual na gravidez (PSFQ)
}

\author{
Translation and validation of the Pregnancy \\ and Sexual Function Questionnaire (PSFQ)
}

\section{Resumo}

OBJETIVO: Adaptar o Pregnancy and Sexual Function Questionnaire (PSFQ) para uso no Brasil, além de avaliar suas propriedades psicométricas. MÉTODOS: Foi realizado um estudo de adaptação e validação com mulheres no último trimestre de gestação, residentes em Rio Branco, Acre. $\bigcirc$ questionário foi traduzido para a língua portuguesa, revisado e avaliado por um grupo de especialistas, tendo sido realizado um pré-teste. A validação do constructo do instrumento foi avaliada por análise fatorial, a consistência interna, pelos coeficientes alfa de Cronbach e ômega de McDonald e a reprodutibilidade, pela estatística kappa por teste-reteste em uma amostra das gestantes. RESULTADOS: A análise fatorial demonstrou a presença de seis domínios: subjetividade, dor e desconforto; frequência e receptividade; desejo; satisfação; orgasmo e estímulo. A consistência interna segundo o alfa foi de 0,6, enquanto na análise do ômega foi de 0,7. Na amostra, o valor de kappa foi superior a 0,7 em todas as questões. CONCLUSÃO: A versão em português do PSFQ pode ser utilizada para avaliar a função sexual durante a gravidez.

\section{Abstract}

PURPOSE: To adapt the Pregnancy and Sexual Function Questionnaire (PSFQ) for use in Brazil and to evaluate its psychometric properties. METHODS: An adaptation and validation study was performed with women in the last trimester of pregnancy living in Rio Branco, Acre. The questionnaire was translated into Portuguese, reviewed and evaluated by specialists, and a pretest was carried out. Construct validity was evaluated by factor analysis; internal consistency was estimated by Cronbach's alpha coefficient and MacDonald's omega, and reproducibility was evaluated by the kappa statistics and testretest in a sample of pregnant women. RESULTS: Factor analysis identified the following six domains: subjectivity, pain and discomfort; frequency and receptivity; desirability; satisfaction; orgasm; and stimulus. The internal consistency by Cronbach's alpha was 0.6, while MacDonald's omega was 0.7 . The kappa value was higher than 0.7 in all questions. CONCLUSION: The Portuguese version of the PSFQ was considered to be adequate for evaluating sexual function during pregnancy.

Correspondêncio

Thatiana Lameira Maciel Amaral Centro de Ciências da Saúde e do Desporto, Campus Universitário BR 364, km 04 - Distrito Industric Caixa Postal 500 (EP: 69920-900

Rio Branco (AC), Brasil

Recebido

$25 / 06 / 2013$

Aceito com modificacõoes $06 / 02 / 2014$
Centro de Ciências da Saúde e do Desporto da Universidade Federal do Acre - UFAC - Rio Branco (AC), Brasil.

'Curso de Enfermagem, Universidade Federal do Acre - UFAC - Rio Branco (AC), Brasil.

${ }^{2}$ Escola Nacional da Saúde Pública da Fundação Oswaldo Cruz - FIOCRUZ - Rio de Janeiro (RJ), Brasil. 


\section{Introdução}

As inúmeras pesquisas na área da saúde e a facilidade de intercâmbio das informações metodológicas contribuem para a necessidade de adaptação transcultural e avaliação das propriedades psicométricas dos instrumentos de medidas oriundos de outros países ${ }^{1}$. Tal avaliação permite determinar se o instrumento é adequado ao que ele se propõe a mensurar, contemplando as dimensões do fenômeno observado e inclui as análises de sua validade e confiabilidade, que são etapas fundamentais para a equivalência de uma medida ${ }^{2}$.

A validação de um instrumento tem como objetivo verificar sua capacidade de medir exatamente aquilo para que foi construído ${ }^{3-5}$. Já o termo confiabilidade refere-se ao grau de concordância entre múltiplas medidas de um mesmo objeto, assim como ao grau de consistência interna entre os seus itens, apontando sua fidedignidade e precisão, ${ }^{5,6}$.

Considera-se como disfunção sexual a ocorrência de qualquer alteração em uma das fases do ciclo da resposta sexual que se manifeste de forma persistente e recorrente ${ }^{7}$, sendo um importante problema de saúde pública devido à sua prevalência e às inúmeras alterações na qualidade de vida da mulher ${ }^{8}$.

Os estudos sobre a resposta sexual mostram que existem diferenças entre homens e mulheres e que instrumentos embasados no modelo linear (desejo, excitação, orgasmo e resolução) hoje são contestados, pois os trabalhos mais recentes apontam que tal resposta não é linear ${ }^{9}$. Assim, no âmbito das disfunções sexuais femininas, a utilização de instrumentos que contemplem questões subjetivas é de grande relevância e usual em países desenvolvidos ${ }^{10,11}$. Entretanto, no Brasil, tais instrumentos ainda são escassos ${ }^{12}$.

Durante a gravidez, a função sexual pode se manifestar de diferentes formas, o que possibilita enriquecer ou reduzir o prazer a dois ${ }^{13}$. Apesar de a gestação advir da sexualidade, estudos apontam a influência da primeira sobre a última por diversos fatores, tais como a alteração na imagem corporal, a mudança de papéis sociais, a qualidade do relacionamento, as alterações hormonais, entre outros ${ }^{14-16}$.

A revisão da literatura científica identificou três instrumentos validados para avaliar os efeitos da gravidez sobre a função sexual no Brasil. O Female Sexual Function Index (FSFI) e o Quociente Sexual Feminino (QSF) foram desenvolvidos para avaliar a função sexual feminina, sendo autoadministrados e não específicos para a gravidez ${ }^{17-19}$. O terceiro, Inventário da Resposta Sexual na Gestação (PSRI), foi construído para gestantes brasileiras, mas não aborda os diferentes períodos gestacionais, nem apresenta os resultados das análises psicométricas ${ }^{20}$.

Nesse contexto, surge a necessidade de se contar com um instrumento que possibilite a avaliação dos domínios da resposta sexual feminina antes e durante a gravidez, aplicável em uma única entrevista a gestantes de diferentes níveis de escolaridade e com capacidade de obter informações sobre a prática sexual e sobre as mudanças na avaliação que a mulher faz sobre si e o seu corpo.

O censo brasileiro de 2010 revelou que $9,6 \%$ da população brasileira com 15 anos ou mais é analfabeta, sendo a maior proporção na região Nordeste $(17,6 \%)$, seguida pela Norte $(10,6 \%)$. No Acre, $15 \%$ da população com idade superior a 15 anos são analfabetos, com variabilidade de 12 a $62 \%$ nos diferentes municípios ${ }^{21}$. Esses dados apontam a importância de instrumentos que sejam aplicados por meio de entrevistas e que captem informações de diferentes momentos da gestação em uma única entrevista, uma vez que a disfunção sexual pode se apresentar a qualquer tempo.

A busca na literatura internacional, realizada pela equipe do projeto "Saúde sexual e reprodutiva de primigestas: análise de fatores relacionados ao tipo de parto", identificou o Pregnancy and Sexual Function Questionnaire (PSFQ), desenvolvido por Gökyildiz e Beji ${ }^{22}$, que contempla as cinco fases da resposta sexual saudável descritos por Basson et al. ${ }^{9}$ :

1. início da atividade sexual, com ou sem a consciência do desejo;

2. excitação subjetiva com respectiva resposta física;

3. sensação de excitação subjetiva, desencadeado a consciência do desejo;

4. aumento gradativo da excitação e do desejo, atingido ou não o orgasmo;

5. satisfação física e emocional, resultando em receptividade para futuros atos.

A prevalência de disfunção sexual em Rio Branco, Estado do Acre, foi de 23,9\% antes da gravidez e de $67,7 \%$ na gravidez ${ }^{23}$. Por entender que a gravidez é um período de intensas transformações, inclusive quanto à sexualidade, desenvolveu-se o presente estudo com o objetivo de realizar a adaptação transcultural e a avaliação das propriedades psicométricas do PSFQ, quanto à confiabilidade e à validade para uso no Brasil.

\section{Métodos}

Trata-se de um estudo metodológico, que seguiu o processo proposto por Beaton et al. ${ }^{24}$ para alcançar a adaptação transcultural, além de validar uma versão brasileira do instrumento PSFQ em gestantes no município de Rio Branco, Acre.

\section{Instrumento}

O PSFQ é um instrumento que pretende avaliar as modificações na função sexual durante a gravidez, 
tendo sido parcialmente publicado no artigo The effects of pregnancy on sexual life $e^{22}$. $\mathrm{O}$ instrumento original foi aplicado a gestantes atendidas no ambulatório de pré-natal da Universidade de Istambul, Turquia. É composto por 27 questões que se relacionam com a ideia geral sobre sexo durante a gravidez, a percepção do corpo, a vida íntima do casal, a frequência de relações sexuais, o desejo e a satisfação sexual, a lubrificação vaginal e a dispareunia. $\mathrm{O}$ instrumento completo foi cedido pelas autoras.

Conforme a versão original, o tempo verbal deve ser adaptado para contemplar os períodos anteriores à gestação, primeiro, segundo e terceiro trimestres nas questões de 12 a 27 . Assim, para cada uma dessas perguntas, existem quatro respostas, correspondendo aos quatro períodos avaliados.

Além do instrumento a ser validado, a entrevista incluiu itens e questões que possibilitaram caracterizar o perfil socioeconômico das gestantes.

A autorização para a tradução foi concedida pelas autoras do instrumento e o projeto de pesquisa foi aprovado pelo Comitê de Ética em Pesquisa da Universidade Federal do Acre (UFAC).

\section{Adaptação transcultural}

Os passos metodológicos para alcançar a equivalência semântica, segundo Beaton et al. ${ }^{24}$, incluem: tradução, retradução (back translation), revisão da tradução por grupo de especialistas e pré-teste.

A tradução do instrumento foi realizada por dois profissionais com fluência em inglês e que tinham como língua materna o português do Brasil. Apenas um estava ciente dos objetivos e conceitos que envolvem o instrumento. Essa etapa resultou em duas traduções (T1 e T2), sendo as ambiguidades solucionadas pelas autoras do presente artigo, resultando em uma síntese dessas duas versões (T12).

Em seguida, a síntese da tradução para o português foi vertida para o inglês por dois outros tradutores bilíngues, independentes, que não receberam informações sobre os conceitos e propósitos do instrumento, resultando em duas retraduções (RT1 e RT2). Foi, então, realizada a comparação dessas versões com o instrumento original em inglês quanto ao significado e à equivalência semântica entre elas. Para apreciação do significado geral, utilizou-se a proposta de Reichenheim et al..$^{25}$ de qualificação em quatro níveis: inalterado, pouco alterado, muito alterado ou completamente alterado. Ao final dessa etapa, chegou-se a uma versão final da retradução (RT12), que foi aprovada pelas autoras da versão original do PSFQ.

A avaliação por um grupo de especialistas, formado por profissionais da área a ser pesquisada pelo instrumento, consolidou a tradução para o português. Ao grupo foi solicitada a avaliação dos itens quanto às equivalências semântica, idiomática, cultural e conceitual, com objetivo de assegurar a validação do conteúdo. A produção de uma versão pré-final do questionário foi alcançada por consenso em relação a cada item.

Após a avaliação pelos especialistas, o instrumento foi aplicado a um grupo de 30 gestantes para realização do pré-teste. Estas estavam no último trimestre e realizavam o pré-natal na Maternidade Bárbara Heliodora ou nos Centros de Saúde Roney Meirelles e Barral y Barral, na zona urbana de Rio Branco. As participantes foram selecionadas de acordo com o agendamento das consultas, no período de dezembro de 2010 a janeiro de 2011 , com idade a partir de 18 anos. Os critérios de exclusão previamente determinados foram: gestantes com comprometimento cognitivo que inviabilizasse a comunicação ou o entendimento das perguntas, as que não tinham parceiro ou aquelas com gravidez de alto risco.

O pré-teste é importante para detectar erros e confirmar se as perguntas são compreensíveis para a população a que se destinam ${ }^{25}$. Também, foram observados os aspectos práticos da aplicação. As alterações propostas pelas entrevistadas para o melhor entendimento do instrumento foram acolhidas, o que resultou na versão final em português (VF).

\section{Propriedades psicométricas}

A população para o estudo das propriedades psicométricas foi uma amostra de conveniência, com gestantes com 35 semanas ou mais de gestação, atendidas nas Unidades de Saúde (Centros de Saúde e Unidades de Saúde da Família) e na maternidade Bárbara Heliodora, residentes na zona urbana de Rio Branco, no período de fevereiro a maio de 2011. Os critérios de exclusão foram os mesmos considerados no pré-teste.

O tamanho mínimo da amostra estimado para validação do instrumento era de 280 gestantes, correspondendo a 10 respondentes por item, considerado adequado para análise fatorial e coeficientes de correlação ${ }^{4}$, tendo sido entrevistadas 352 gestantes.

O teste-reteste foi realizado em 100 gestantes no último trimestre para a avaliação da reprodutibilidade do instrumento. De acordo com a tabulação disponibilizada por Sim e Wright ${ }^{26}$, o número mínimo para avaliação de kappa entre dois observadores, sendo considerada uma proporção de positividade de $50 \%$, um nível de significância de 0,05 e um poder de $90 \%$, seria de 88 participantes; entretanto, sabe-se que aumentar o número de sujeitos é uma estratégia para incrementar a precisão das estimativas.

Após a aprovação pelo Comitê de Ética em Pesquisa da UFAC, foram realizadas visitas às Unidades de Saúde da Família, centros de saúde localizados na zona urbana de Rio Branco, assim como à maternidade Bárbara Heliodora, para aplicação dos questionários às gestantes 
que cumpriam os critérios de inclusão e assinaram o termo de consentimento livre e esclarecido.

Em visita às unidades de saúde, foram obtidas informações sobre os dias e horários de realização do pré-natal. Aquelas com idade gestacional superior a 35 semanas, segundo a data da última menstruação anotada no "Cartão da Gestante", eram convidadas a participar por ordem de chegada ao serviço de saúde, sendo informado o objetivo da pesquisa e o sigilo das respostas. As entrevistas foram realizadas em local reservado, sendo todas as perguntas feitas diretamente à gestante.

Após a aplicação do questionário, foram mantidos contatos semanais por telefone com as primeiras 150 gestantes para posterior realização do reteste. Esse total foi escolhido com o intuito de alcançar pelo menos 100 gestantes, devido à possibilidade de perdas de seguimento.

A entrevista do reteste foi realizada por um único avaliador, diferente daquele que realizou a primeira entrevista, dentro do intervalo de 15 a 20 dias da primeira aplicação, no período do pós-parto imediato, durante a internação. A escolha do local facilitou a coleta das informações por ser a única maternidade em funcionamento no período do estudo, em Rio Branco. Ao conseguir a segunda entrevista de 100 gestantes, as demais foram informadas do término da coleta para o reteste.

$\mathrm{Na}$ análise dos dados, foi utilizada a estatística descritiva para a caracterização da amostra por meio de média e desvio-padrão das variáveis contínuas e proporções das categóricas. As questões abertas do PSFQ foram categorizadas em conformidade com as respostas das entrevistadas, permanecendo no estudo como: sentimento quanto às mudanças no corpo (feliz, indiferente/normal, triste); pensamento sobre o que o parceiro acha do seu corpo (bonito, indiferente/normal, feio); roupas de grávida tornam as mulheres atraentes (sim, não); e tempo para ter a primeira relação sexual após saber que estava grávida (não manteve relações sexuais, mais de um mês, de duas a quatro semanas, na mesma semana, no mesmo dia).

Em relação à confiabilidade, foi utilizado o kappa ponderado e respectivo intervalo de confiança de $95 \%$ (IC95\%) para avaliar a estabilidade do instrumento (reprodutibilidade) ao medir a proporção de concordância observada entre as duas avaliações em um mesmo indivíduo, sendo levado em conta o acaso. Para sua classificação, foram utilizados os pontos de corte propostos por Landis e $\mathrm{Koch}^{27}$ : 0 a 0,20 (fraca); 0,21 a 0,40 (razoável); 0,41 a 0,60 (moderada); 0,61 a 0,80 (substancial); 0,81 a 0,99 (quase perfeita); e 1 (perfeita).

A análise da consistência interna foi avaliada pelos coeficientes alfa de Cronbach e ômega de McDonald, tendo sido considerado como limite inferior aceitável valores acima de $0,60^{4}$. O alfa e o ômega são estimados com base em relações bisseriais (entre variáveis ordinais e contínuas) e policóricas (variáveis ordinais). A utilização desses dois indicadores deve-se ao fato de o alfa ser considerado o menor e o ômega, o maior valor atribuível à consistência interna, sendo o cálculo baseado na proporção da variância comum dos itens do instrumento ${ }^{28-30}$.

A validade do instrumento foi avaliada pela análise fatorial que tem como objetivo identificar relações inaparentes entre os itens, agrupando aqueles correlacionados entre si. Para avaliar o ajuste dos dados para essa técnica de análise estatística, foram usados o índice Kaiser-MeyerOlkin e o teste de esfericidade de Bartlett. Para a análise dos componentes principais, foram escolhidos os fatores que obtiveram autovalores (eigenvalues) superiores a 1 . A extração dos fatores principais foi efetuada após rotação Varimax e critério de Kaiser.

O processamento e a análise dos dados foram realizados no programa Statistical Package for the Social Sciences (SPSS), versão 13, para Windows. A análise do ômega de McDonald foi realizada na rotina psych do $\mathrm{R}$ e a da estatística kappa, no STATA, versão 10.

\section{Resultados}

Os domínios da função sexual, contidos na versão original do PSFQ, foram considerados adequados para sua utilização no Brasil. Eles abarcam as alterações da vida sexual ocorridas durante a gravidez, na avaliação dos profissionais da área envolvidos nesse estudo e estão em consonância com o que se encontra na literatura científica. Com a equivalência do conteúdo função sexual para o contexto brasileiro, verificou-se que os itens do questionário representavam os domínios necessários para avaliação do referido constructo.

As divergências nas retraduções foram solucionadas, o que possibilitou a produção da versão pré-final pelos especialistas, sendo observados os aspectos estilísticos e de clareza dos termos. Posteriormente, a participação das gestantes no pré-teste resultou na inclusão de termos coloquiais na versão final, com o intuito de facilitar sua compreensão junto à população. Vale ressaltar que esses termos não substituíram os técnico-científicos, mas foram acrescentados como opção para facilitar o entendimento das perguntas. A versão final da tradução foi a resultante da apreciação do grupo de especialistas e das participantes do pré-teste, estando o questionário disponível no link: https://drive. google.com/file/d/0B5kOlY9DyOcfWTAtLUVKMIRvZE0/ edit? usp $=$ sharing

Em relação às 352 gestantes entrevistadas para a análise da validação, a idade variou entre 15 e 35 anos, com média de 23,9 anos (desvio-padrão-DP 4,4). Acima de 90\% delas relatou situação conjugal estável e $66,7 \%$ referiam ensino médio ou superior. A maioria não tinha renda própria e contava com renda familiar inferior a dois salários mínimos. $\mathrm{O}$ início da vida sexual ocorreu em média aos 15 anos de idade (DP 2,1). Mais de $40 \%$ das entrevistadas eram primigestas (Tabela 1). 


\section{Confiabilidade}

A estatística kappa foi calculada para as questões ordinais do PSFQ, sendo excluídas sete questões por apresentarem caselas de valor igual a zero no teste-reteste. As questões excluídas e o percentual de concordância foram: "1. Esta gravidez foi desejada? (99,0\%); 2.2. Você teve dificuldade em engravidar, mesmo querendo? $(98,0 \%)$; 3. Se sim, realizou tratamento para infertilidade (tratamento para engravidar) antes desta gravidez? $(100,0 \%)$; 4. Se realizou, isto influenciou na vida sexual? $(100,0 \%) ; 8$. Você teve algum problema durante a gravidez? $(90,0 \%)$; 23. Em quais destas opções você experimenta o orgasmo ("clímax")? (99,0\%); 24. Quais as posições que você adotou durante a gravidez? (91,0\%)”.

Tabela 1. Caracteristicas socioeconômicas e reprodutivas das gestantes entrevistadas em Rio Branco, Acre

\begin{tabular}{|c|c|c|}
\hline Variáveis & $\mathrm{n}$ & $\%$ \\
\hline \multicolumn{3}{|l|}{ Idade (anos) } \\
\hline 15 a 20 & 89 & 25,3 \\
\hline 21 a 25 & 144 & 40,9 \\
\hline 26 a 30 & 90 & 25,6 \\
\hline 31 a 35 & 29 & 8,2 \\
\hline Média $\pm D P$ & \multicolumn{2}{|c|}{$23,9 \pm 4,4$} \\
\hline \multicolumn{3}{|l|}{ Siłuação conjugal } \\
\hline Casada & 73 & 20,7 \\
\hline Vive com companheiro & 263 & 74,7 \\
\hline $\begin{array}{l}\text { Tem companheiro, mas não } \\
\text { vive com ele ou não é fixo }\end{array}$ & 16 & 4,6 \\
\hline \multicolumn{3}{|l|}{ Escolaridade } \\
\hline Nunca estudou & 07 & 2,0 \\
\hline Ensino Fundamental & 110 & 31,3 \\
\hline Ensino Médio & 203 & 57,7 \\
\hline Ensino Superior & 32 & 9,0 \\
\hline \multicolumn{3}{|l|}{ Renda própria* } \\
\hline Sim & 111 & 31,8 \\
\hline Não & 238 & 68,2 \\
\hline \multicolumn{3}{|c|}{ Renda familiar" (Salários mínimos) } \\
\hline$\leq 1$ & 107 & 30,7 \\
\hline De 1 a $<2$ & 103 & 29,5 \\
\hline De $2 a<5$ & 132 & 37,8 \\
\hline$\geq 5$ & 07 & 2,0 \\
\hline \multicolumn{3}{|l|}{ Sexarca (anos) } \\
\hline$<15$ & 150 & 42,6 \\
\hline Igual a 15 & 115 & 32,7 \\
\hline$>15$ & 87 & 24,7 \\
\hline Média $\pm D P$ & \multicolumn{2}{|c|}{$15,0 \pm 2,1$} \\
\hline \multicolumn{3}{|l|}{ Gestações } \\
\hline 1 & 143 & 40,6 \\
\hline De 2 a 4 & 172 & 48,9 \\
\hline$\geq 5$ & 37 & 10,5 \\
\hline Média $\pm D P$ & \multicolumn{2}{|c|}{$2,4 \pm 1,6$} \\
\hline Total & 352 & 100,0 \\
\hline
\end{tabular}

*n menor na renda própria e na renda familiar.
A amostra para a análise da confiabilidade foi de 100 gestantes. Os valores de kappa foram superiores a 0,85 , configurando uma concordância, excluído o acaso quase perfeito, segundo a classificação de Landis e $\mathrm{Koch}^{27}$ para todas as questões, exceto a $7^{\mathrm{a}}$, referente à opinião do parceiro sobre o corpo da gestante, classificada como substancial, com kappa de 0,73 (Tabela 2).

A consistência interna, avaliada pelo coeficiente alfa de Cronbach, para o instrumento obteve o resultado de 0,64 , enquanto a avaliação pelo ômega de McDonald foi superior, totalizando 0,74 .

\section{Validade}

Com base na análise fatorial por componentes principais, das 28 questões do PSFQ, foram obtidos 6 fatores, assim nomeados:

1. frequência e receptividade;

2. desejo;

3. subjetividade, dor e desconforto;

4. satisfação;

5. estímulo;

6. orgasmo.

Duas questões ( 9 e 25 ) pontuaram em mais de um domínio, ambas se relacionaram com o domínio 5, tendo mantido um valor próximo de 0,4 (Tabela 3).

Tabela 2. Concordância e coeficiente kappa obtido pelo teste-reteste das questões mantidas do Pregnancy and Sexual Function Questionnaire

\begin{tabular}{|c|c|c|}
\hline Itens do PSFQ & Kappa & IC95\% \\
\hline 05. 0 que você acha sobre sexo durante a gravidez? & 0,9 & $0,9-0,9$ \\
\hline 07. 0 que seu parceiro acha sobre seu corpo? & 0,7 & $0,6-0,7$ \\
\hline 09. Com que frequência você tinha relação sexual? & 0,9 & $0,9-0,9$ \\
\hline 10. Com que frequência você sentia desejo sexual? & 0,9 & $0,8-0,9$ \\
\hline 11. Quem tinha a iniciativa para relação ou atividade sexual? & 0,9 & $0,9-0,9$ \\
\hline $\begin{array}{l}\text { 12. Como você pensa que seu parceiro considera a relação } \\
\text { sexual de vocês dois? }\end{array}$ & 0,9 & $0,9-0,9$ \\
\hline $\begin{array}{l}\text { 13. Em geral, quão satisfatória é para você sua relação sexual } \\
\text { com seu parceiro? }\end{array}$ & 0,9 & $0,8-0,9$ \\
\hline $\begin{array}{l}\text { 14. Quando seu parceiro toma iniciativa na relação sexual de } \\
\text { vocês, como você geralmente responde? }\end{array}$ & 0,8 & $0,8-0,9$ \\
\hline 15. Você teve secura vaginal que dificultusse a relação sexual? & 0,8 & $0,7-0,9$ \\
\hline 16. Você tem dor genital durante 0 ato sexual? & 0,8 & $0,8-0,9$ \\
\hline $\begin{array}{l}\text { 19. Quando você teve a sua primeira relação sexual depois de } \\
\text { saber que estava grávida? }\end{array}$ & 0,9 & $0,8-0,9$ \\
\hline 20. Você se sente estimulada durante a relação sexual? & 0,8 & $0,8-0,9$ \\
\hline 21. Quanto tempo duram as preliminares? & 0,9 & $0,9-0,9$ \\
\hline 22. Quanto tempo dura o ato sexual? & 0,8 & $0,7-0,8$ \\
\hline 25. Você tem medo de manter relações sexuais? & 0,8 & $0,8-0,8$ \\
\hline $\begin{array}{l}\text { 26. Seu parceiro já experimentou episódio de } \\
\text { não manter a ereção? }\end{array}$ & 0,8 & $0,8-1,0$ \\
\hline
\end{tabular}

PSFQ: Pregnancy and Sexual Function Questionnaire. 
Tabela 3. Cargas fatoriais dos itens mantidos no modelo do Pregnancy and Sexual Function Questionnaire

\begin{tabular}{|c|c|c|c|c|c|c|c|}
\hline \multicolumn{2}{|c|}{ Itens do PSFQ" } & \multicolumn{6}{|c|}{ Componentes } \\
\hline $\mathrm{N}^{0}$ & Descrição & Frequência e receptividade & Desejo & Subjetividade, dor e desconforto & Satisfação & Estímulo & Orgasmo \\
\hline 22 & Tempo de duração do ato sexual & 0,7 & & & & & \\
\hline 21 & Tempo de duração das preliminares & 0,7 & & & & & \\
\hline 14 & Resposta à iniciativa do parceiro & 0,5 & & & & & \\
\hline 11 & Iniciativa para relação sexual & 0,5 & & & & & \\
\hline 05 & Percepç̃o sobre sexo na gravidez & & 0,6 & & & & \\
\hline 09 & Frequência de relação sexual & & 0,5 & & & 0,4 & \\
\hline 16 & Dor genital durante 0 ato sexual & & & 0,7 & & & \\
\hline 26 & Não manutenção da ereção pelo parceiro & & & 0,7 & & & \\
\hline 15 & Secura vaginal & & & 0,4 & & & \\
\hline 25 & Medo de manter relações sexuais & & & 0,4 & & 0,4 & \\
\hline 07 & Percepção da avaliação do parceiro sobre o corpo da gestante & & & & & & 0,7 \\
\hline 23 & Orgasmo & & & & & & 0,6 \\
\hline
\end{tabular}

\#As questões referentes aos itens estão disponíveis no link: https://drive.google.com/file/d/OB5kOlY9DyOcfWTAtLUVKMIRvZEO/edit? usp=sharing

Método de extração pela análise do componente principal e de rotação por varimax com normalização Kaiser. KMO=0,692; $\chi^{2}=865,597 ; p<0,001$.

PSFQ: Pregnancy and Sexual Function Questionnaire.

A análise inicial dos autovalores dos 6 domínios, após rotação, explicou $57,96 \%$ da variância. Os valores de comunalidades variaram entre 0,46 a 0,73 , sendo que as questões com menores comunalidades foram "O que você acha sobre sexo durante a gravidez?” (0,49), "Quando seu parceiro toma iniciativa na relação sexual de vocês, como você geralmente responde?" $(0,48)$ e "Quando você teve a sua primeira relação sexual depois de saber que estava grávida?" $(0,46)$. Assim, como a maioria das questões apresentou altas comunalidades, os seis fatores extraídos são capazes de explicar a variância comum dos indicadores.

\section{Discussão}

O modelo utilizado, proposto por Beaton et al. ${ }^{24}$, demonstrou coerência dos passos metodológicos que visam garantir a veracidade e aplicabilidade do instrumento. Ele mostrou ser um bom método para obtenção da adaptação transcultural, visto que possibilitou o fornecimento de elementos para a manutenção da similaridade entre as versões e facilitou a tomada de decisões para a elaboração da uma versão-síntese com similaridade linguística.

Após a adaptação, foi avaliada a análise das propriedades psicométricas do PSFQ, que revelou ser uma ferramenta útil para identificar alterações na função sexual feminina durante a gravidez. Identificar tais mudanças é importante para a manutenção da saúde geral e da qualidade de vida da mulher e do casal ${ }^{7}$.

Os resultados do kappa ponderado, após a aplicação do teste-reteste, variaram de 0,73 a 1,00, com alta concordância, comprovando sua estabilidade. Esses resultados sugerem não haver diferença significativa entre a aplicação do instrumento no final da gravidez e no pós-parto imediato, fato que viabiliza seu emprego na maternidade para avaliar a função sexual durante a gestação. A menor concordância foi observada na pergunta sobre o que a gestante pensa que o parceiro acha do seu corpo grávido (kappa - k=0,73; IC95\% 0,65-0,76). Uma hipótese para esse resultado é o reteste ter sido realizado no pós-parto imediato, quando o corpo da mulher mudou completamente em virtude do nascimento da criança.

Outra medida da confiabilidade é a consistência interna, responsável por avaliar a correlação entre as variáveis em uma escala múltipla ${ }^{4,31}$. No presente estudo, o ômega de McDonald foi de 0,74 para o PSFQ, acima do valor observado para o alfa de Cronbach $(0,64)$. Essa discordância nesses índices também foi observada no estudo realizado para validar o Índice de Capacidade para o Trabalho (ICT) em trabalhadores de enfermagem ${ }^{32}$. Assim, optou-se por informar os dois resultados como uma forma de mostrar a variabilidade mínima e máxima da consistência interna do instrumento.

A forma de cálculo mais comum para essa análise é o alfa de Cronbach, que depende do número de itens da escala, podendo ser diretamente aumentado por esse fator ${ }^{33}$. Segundo o próprio Cronbach, o alfa não consegue expressar todas as informações da confiabilidade, sendo visto como uma pequena medição do todo que representa essa análise ${ }^{34}$.

Para a validação do instrumento, optou-se pela utilização da técnica estatística multivariada de análise fatorial. Os seis fatores encontrados pela análise do componente principal do PSFQ compreendem os domínios necessários para avaliar a 
função sexual feminina, com base nas etapas da resposta sexual saudável das mulheres. É ressaltada a relevância de perguntas subjetivas que permeiam o universo feminino quando o assunto é sexo, questões que permitam não apenas mensurar $\mathrm{o}$ ato, mas o que acontece antes, durante e depois dele.

Nos resultados da análise fatorial realizada, a presença de 2 questões ( 9 e 25) que pontuaram em diferentes domínios pode ser explicada pela influência que a frequência da relação sexual e o medo de realizá-las tem no contexto da função sexual feminina durante a gravidez. A frequência é relacionada com o estímulo e com o desejo, bem como o medo influencia no estímulo e na subjetividade, dor e desconforto na relação sexual. Apesar de apresentarem valores menores no domínio estímulo, as questões ressaltam o dinamismo da função sexual feminina durante a gestação.

Os instrumentos validados no Brasil para avaliar a função sexual não utilizaram esse método. O FSFI utilizou a correlação de Pearson em comparação com o Quociente Sexual - versão feminina (QS-F), em uma amostra de 32 gestantes. Os domínios excitação, lubrificação, orgasmo e satisfação do FSFI apresentaram fortes correlações com os domínios do desejo e interesse sexual, preliminares, excitação pessoal e sintonia com o parceiro, orgasmo e satisfação, não tendo correlação com o domínio conforto/dor ${ }^{17}$.

O PSRI apresenta como domínios a frequência, o desejo, a excitação, o orgasmo, o prazer, a dispareunia, a iniciação e as dificuldades sexuais femininas e do parceiro, tendo realizado apenas a validação de conteúdo por meio da avaliação de especialistas ${ }^{20}$.

Os três instrumentos apresentados são amplamente utilizados no Brasil ${ }^{35-37}$; no entanto, não abordam todas as questões que permeiam a função sexual feminina na gestação, fato confirmado na análise fatorial do PSFQ ao incluir no domínio orgasmo a percepção da imagem corporal. Estudo prospectivo realizado com gestantes confirmou a associação entre imagem corporal e função sexual ${ }^{38}$.

No entanto, uma limitação do presente estudo foi a ausência dos resultados das análises psicométricas do instrumento original, para comparação. Entretanto, o instrumento foi escolhido por ter a capacidade de avaliar a função sexual feminina em seus diferentes domínios, por abordar os diferentes trimestres da gestação e pelo método de aplicação.
Outro potencial limite foi a entrevista face-a-face, que pode ser um fator de constrangimento, sendo tal risco controlado ao serem feitas as perguntas em local reservado, porém tem a vantagem de não depender do nível de escolaridade das entrevistadas. Em estudo realizado em Maceió, com 92 gestantes, um dos critérios de inclusão era ter cursado no mínimo até a $5^{\text {a }}$ série do ensino fundamental, uma vez que o instrumento utilizado era autoaplicável ${ }^{17}$. No Brasil, a aplicação de tal critério excluiria da pesquisa um grande contingente de mulheres, o que inviabilizaria a descoberta de alterações na função sexual de forma igualitária.

Por outro lado, o presente trabalho explicita as diversas etapas a serem realizadas no processo de adaptação transcultural com o intuito de divulgar uma experiência bem sucedida e traz uma importante contribuição ao disponibilizar um instrumento validado para avaliar os efeitos da gestação sobre a função sexual feminina. Além disso, o PSFQ aborda questões não mencionadas em outros instrumentos, tais como frequência de coito e percepção sobre a imagem corporal.

Assim, a tradução e adaptação transcultural do PSFQ para a população brasileira foram concluídas de acordo com a metodologia adotada. A versão pré-testada mostrou-se compreensível e de fácil aplicação. Os resultados obtidos na análise da consistência interna e da reprodutibilidade confirmam a confiabilidade do instrumento para uso em gestantes no Brasil, podendo sua aplicação ser realizada na gestação ou no pós-parto imediato sem prejuízo da reprodutibilidade. A validação do PSFQ, por análise fatorial, demonstrou a presença de seis domínios condizentes com a literatura sobre a temática. Portanto, a versão em português do PSFQ possui propriedades psicométricas adequadas, sendo configurada em um instrumento útil para avaliar a função sexual durante a gravidez.

\section{Agradecimentos}

À Coordenação de Aperfeiçoamento de Pessoal de Nível Superior (CAPES); ao Programa de Pós-graduação em Saúde Pública e Meio Ambiente da Escola Nacional de Saúde Pública da Fundação Oswaldo Cruz (ENSP/ FIOCRUZ); à Fundação de Tecnologia do Estado do Acre (FUNTAC) e ao colaborador Sérgio Henrique Almeida da Silva Júnior, doutorando da ENSP/FIOCRUZ.

\section{Referências}

1. Reichenheim ME, Moraes CL. Operacionalização de adaptação transcultural de instrumentos de aferição usados em epidemiologia. Rev Saúde Pública. 2007;41 (4):665-73.

2. Fayers PM, Machin D. Quality of life: the assessment, analysis and interpretation of patientreported outcomes. 2nd ed. Hoboken: Wiley; 2007.

3. Fletcher RH, Fletcher SW. Epidemiologia clínica: elementos essenciais. 4a ed. Porto Alegre: Artmed; 2006.
4. Hair JF Jr, Black WC, Babin BJ, Anderson RE, Tatham RL. Análise multivariada de dados. 6a ed. Porto Alegre: Artmed; 2009.

5. McDowell I. Measuring health: a guide to rating scales and questionnaires. 3rd ed. New York: Oxford University Press; 2006.

6. Streiner DL, Norman GR. Health measurement scales: a practical guide to their development and use. 4th ed. New York: Oxford University Press; 2008. 
7. Abdo CHN, Fleury HJ. Aspectos diagnósticos e terapêuticos das disfunções sexuais femininas. Rev Psiquiatr Clín. 2006;33(3): 162-7.

8. World Health Organization. Defining sexual health: report of a technical consultation on sexual health, 28-31 January 2002, Geneva. Geneva: WHO; 2006.

9. Basson R, Leiblum S, Brotto L, Derogatis L, Fourcroy J, Fugl-Meyer K, et al. Revised definitions of women's sexual dysfunction. J Sex Med. $2004 ; 1(1): 40-8$.

10. Daker-White $G$. Reliable and valid self-report outcome measures in sexual (dys)function: systematic review. Arch Sex Behav. 2002;31(2): 197-209.

11. Pasqualotto EB, Pasqualotto FF, Sobreiro, BP, Lucon AM. Female sexual dysfunction: the important points to remember. Clinics (Sao Paulo). 2005;60(1):51-60.

12. Valadares ALR, Pinto-Neto AM, Sousa MH, Osis MD. [Sociocultural adaptation of the short personal experiences questionnaire (SPEQ) in Brazil]. Rev Bras Ginecol Obstet. 2010;32(2):72-6. Portuguese.

13. Lech MB, Martins PCR. Oscilações do desejo sexual no período gestacional. Estud Psicol (Campinas). 2003;20(3):37-46.

14. De Judicibus MA, McCabe MP. Psychological factors and the sexuality of pregnant and postpartum women. J Sex Res. 2002;39(2):94-103.

15. LaMarre AK, Paterson LQ, Gorzalka BB. Breastfeeding and postpartum maternal sexual functioning: a review. Can J Hum Sex. 2003;12(3-4):151-68.

16. Savall ACR, Mendes AK, Cardoso FL. Perfil do comportamento sexual na gestação. Fisioter Mov. 2008;21 (2):61-70.

17. Leite APL, Moura EA, Campos AAS, Mattar R, Souza E, Camano L. [Validation of the female sexual function index in Brazilian pregnant women]. Rev Bras Ginecol Obstet. 2007;29(8):396401. Portuguese.

18. Rosen R, Brown C, Heiman J, Leiblum S, Meston C, Shabsigh R, et al. The Female Sexual Function Index (FSFI): a multidimensional self-report instrument for the assessment of female sexual function. J Sex Marital Ther. 2000;26(2):191-208.

19. Abdo CHN. Quociente sexual feminino: um questionário brasileiro para avaliar a atividade sexual da mulher. Diagn Tratamento. 2009; 14(2):89-91.

20. Rudge CVC, Calderon IMP, Dias A, Lopes GP, Barbosa AP, Maestá I, et al. Design and validity of a questionnaire to assess sexuality in pregnant women. Reprod Health. 2009;6:12.

21. Instituto Brasileiro de Geografia e Estatística. Comunicação Social [Internet]. Indicadores sociais municipais 2010: incidência de pobreza é maior nos municípios de porte médio. 2011 [citado 2012 Jan 12]. Disponível em: <http://www.ibge.gov.br/home/presidencia/ noticias/noticia_visualiza.php?iid_noticia $=2019 \&$ \&d_pagina $=1>$.

22. Gökyildiz S, Beji NK. The effects of pregnancy on sexual life. J Sex Marital Ther. 2005;31(3):201-15.
23. Lima AC, Dotto LMG, Mamede MV. Prevalência de disfunção sexual em primigestas, no Município de Rio Branco, Acre, Brasil. Cad Saúde Pública. 2013;29(8):1544-54.

24. Beaton D, Bombardier C, Guillemin F, Ferraz MB. Recommendations for the cross-cultural adaptation of the DASH \& QuickDASH outcome measures [Internet]. Toronto: Institute for Work \& Health; 2007 [cited 2010 May 18]. Available from: http://www.dash. iwh.on.ca/assets/images/pdfs/X-CulturalAdaptation-2007.pdf

25. Reichenheim ME, Moraes CL, Hasselmann MH. Equivalência semântica da versão em português do instrumento "Abuse Assessment Screen" para rastrear a violência contra a mulher grávida. Rev Saúde Pública. 2000;34(6):610-6.

26. Sim J, Wright CC. The kappa statistic reliability studies: use, interpretation, and sample size requirements. Phys Ther. 2005;85(3):257-68.

27. Landis JR, Koch GG. The measurement of observer agreement for categorical data. Biometrics. 1977;33(1):159-74.

28. McDonald RP. Test theory: a unified treatment. Mahwah: Lawrence Erlbaum; 1999.

29. Zinbarg RE, Revelle W, Yovel I, Li W. Cronbach's $\alpha$, Revelle's $\beta$, and Mcdonald's $\omega_{H}$ : their relations with each other and two alternative conceptualizations of reliability. Psychometrika. 2005;70(1):123-33.

30. Revelle W, Zinbarg RE. Coefficients alpha, beta, omega, and the GLB: comments on Sijtsma. Psychometrika. 2009;74(1):145-54.

31. Polit DF, Beck CT, Hungler BP. Fundamentos de pesquisa em enfermagem: métodos, avaliação e utilização. 5a ed. Porto Alegre: Artmed; 2004.

32. Silva Junior SHA, Vasconcelos AGG, Griep RH, Rotenberg L. Validade e confiabilidade do índice de capacidade para o trabalho (ICT) em trabalhadores de enfermagem. Cad Saúde Pública. $2011 ; 27(6): 1077-87$.

33. Cortina JM. What is coefficient alpha? An examination of theory and applications. J Appl Psychol. 1993;78(1):98-104.

34. Cronbach L, Shavelson RJ. My current thoughts on coefficient alpha and successor procedures [Internet]. Los Angeles: The Regents of the University of California; 2004 [cited 2011 Oct 20]. Available from: http://www.cse.ucla.edu/products/reports/r643.pdf

35. Ferreira DQ, Nakamura MU, Souza E, Mariani Neto C, Ribeiro MC, Santana TGM, et al. [Sexual function and quality of life of low-risk pregnant women]. Rev Bras Ginecol Obstet. 2012; 34(9):409-13.

36. Naldoni LM, Pazmiño MA, Pezzan PA, Pereira SB, Duarte G, Ferreira $\mathrm{CH}$. Evaluation of sexual function in Brazilian pregnant women. J Sex Marital Ther. 2011 1;37(2): 1 16-29.

37. Leite APL, Campos AAS, Dias ARC, Amed AM, De Souza E, Camano $L$. Prevalence of sexual dysfunction during pregnancy. Rev Assoc Med Bras. 2009;55(5):563-8.

38. Pauls RN, Occhino JA, Dryfhout VL. Effects of pregnancy on female sexual function and body image: a prospective study. J Sex Med. 2008;5(8): 1915-22. 